\title{
The Combined Effect of Chemical Reaction, Thermal Radiation on steady free convection and mass transfer flow in a porous medium considering Soret and Dufour effects
}

\author{
G.Sreedhar Sarma ${ }^{1}$, K.Rama Krishna Prasad ${ }^{2}$, K.Govardhan ${ }^{3}$ \\ ${ }^{I}$ (Department of Mathematics, CVR College of Engineering,Mangalpalli(V),Hyderabad.A.P.) \\ ${ }^{2}$ (Department of Mathematis, S.V.University, Tirupathi.A.P.) \\ ${ }^{3}$ (Department of Mathematics, GITAM UNIVERSITY, Hyderabad,A.P,India)
}

\begin{abstract}
In the present analysis, we study the two-dimensional, steady, free convection and mass transfer flow past a continuously moving semi infinite vertical porous plate in a porous medium in the presence of thermal radiation, chemical reaction, Dufour and Soret effects. The governing nonlinear partial differential equations have been reduced to the coupled nonlinear ordinary differential equations by the similarity transformations. The resulting equations are then solved numerically using finite difference method. The non-dimensional velocity, temperature and concentration profiles are displayed graphically for various interesting parameters entering into the problem and discussed in detail.
\end{abstract}

Key words: Free convection, vertical plate, porous medium, steady flow, Dufour and Soret effects, thermal radiation.

\section{Introduction}

Coupled heat and mass transfer by free convective in a porous media has been widely studied in the recent years due to its wide applications in engineering as postaccidental heat removal in nuclear reactors, solar collectors, drying processes, heat exchangers, geothermal and oil recovery, building construction, etc. (Nield and Bejan [1], Ingham and Pop [2], Vafai [3], Vadasz [4], etc.). It is well known that conventional heat transfer fluids, including oil, water, and ethylene glycol mixture are poor heat transfer fluids, since the thermal conductivity of these fluids plays an important role on the heat transfer coefficient between the heat transfer medium and the heat transfer surface. Anghel M.et al. [5] studied Dufour and Soret effects on free convection boundary-layer over a vertical surface embedded in a porous medium. Postelnicu A. [6] discussed the Influence of a magnetic field on heat and mass transfer by natural convection from vertical surfaces in porous media considering Soret and Dufour effects.

Thermal radiation in fluid dynamics has become a significant branch of the engineering sciences and is an essential aspect of various scenarios in mechanical, aerospace, chemical, environmental, solar power and hazards engineering. For some industrial applications such as glass production and furnace design and in space technology applications such as cosmical flight aerodynamics rocket, propulsion systems, plasma physics and spacecraft re-entry aerothermodynamics which operate at higher temperatures, radiation effects can be significant. The effect of radiation on MHD flow and heat transfer problem has become more important industrially. At high operating temperatures, radiation effect can be quite significant. Many processes in engineering areas occur at high temperatures and knowledge of radiation heat transfer becomes very important for design of reliable equipment, nuclear plants, gas turbines and various propulsion devices or aircraft, missiles, satellites and space vehicles. Anuar Ishak [7] studied MHD Boundary layer flow due to an exponentially stretching sheet with radiation effect. P.Bala Anki Reddy and N.Bhaskar Reddy [8] discussed the Thermal radiation effects on hydro-magnetic flow due to an exponentially stretching sheet. V.Singh and Shweta Agarwal [9] studied the Heat transfer in a second grade fluid over an exponentially stretching sheet through porous medium with thermal radiation and elastic deformation under the effect of magnetic field. Makinde,O.D. and A. Ogulu [10] studied the effect of thermal radiation on the heat and mass transfer flow of a variable viscosity fluid past a vertical porous plate permeated by a transverse magnetic field. S Shivaiah and J Anand Rao [11] discussed the Effects of Soret Dufour and thermal radiation on unsteady MHD free convection flow past an infinite vertical porous plate in the presence of chemical reaction. S. R. VEMPATI and A. B. LAXMINARAYANA-GARI [12] studied Soret and Dufour effects on unsteady MHD flow past an infinite vertical porous plate with thermal radiation. Shateyi, S. and Petersen, M [13] studied the thermal radiation and buoyancy effects on heat and mass transfer over a semi-infinite stretching surface with suction and blowing. Chamkha, A.J et al. [14] studied the Radiation effects on free convection flow past a semi- infinite vertical plate with mass transfer. 
Chemical reactions can be codified as either heterogeneous or homogeneous processes. This depends on whether they occur at an interface or as a single-phase volume reaction. A reaction is said to be of first order, if the rate of reaction is directly proportional to the concentration itself. In many chemical engineering processes, there does occur the chemical reaction between a foreign mass and the fluid in which the plate is moving. In view of heat and mass transfer and chemical reactions numerous and wide-ranging applications in various fields like polymer processing industry in particular in manufacturing process of artificial film and artificial fibers and in some applications of dilute polymer solution. Mansour, M. A., et al. [15] studied the effects of chemical reaction and thermal stratification on MHD free convective heat and mass transfer over a vertical stretching surface embedded in a porous media considering Soret and Dufour numbers. B. Chandra and M. Kumar [16] discussed the Combined Effect of Chemical Reaction, Radiation on Heat and Mass Transfer along a Continuously Moving Surface in Presence of Thermophoresis. M.Q. Al-Odat and T.A. Al-Azab [17] studied the Influence of chemical reaction on transient MHD free convection over a moving vertical plate. Ibrahim FS et al. [18] studied the Effect of the chemical reaction and radiation absorption on the unsteady MHD free convection flow past a semi infinite vertical permeable moving plate with heat source and suction. Kesavaiah DCh et al. [19] discussed the Effects of the chemical reaction and radiation absorption on an unsteady MHD convective heat and mass transfer flow past a semi-infinite vertical permeable moving plate embedded in a porous medium with heat source and suction.

Our aim is to extend the work of M.S.Alam et al. [20]. They have neglected the effects of chemical reaction and thermal radiation. The objective of this paper is to analyze the influence of chemical reaction with Soret and Dufour effects on steady free convection and mass transfer flow past a semi-infinite moving vertical plate embedded in a porous medium by taking thermal radiation into account. The governing equations are transformed by using similarity transformation and the resultant dimensionless equations are solved numerically using the finite difference method. The effects of various governing parameters on the velocity, temperature, and concentration are shown in figures and analyzed in detail.

\section{Mathematical Analysis}

A two-dimensional steady free convection and mass transfer flow of a viscous incompressible fluid past a continuously moving semi-infinite vertical porous flat plate in a porous medium is considered. The flow is assumed to be in the $x$-direction which is taken along the plate in the upward direction and the $y$-axis is taken normal to it. The coordinate system and the flow configuration are shown in the following Fig.1.

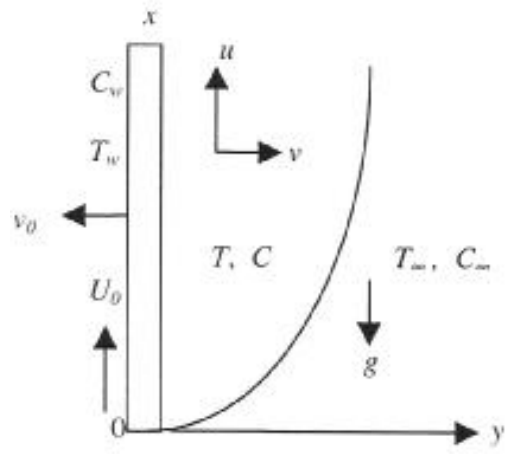

Fig.1: Flow configuration.

Then under the usual Boussinesq's approximation, the governing equations relevant to the problem are:

\section{Continuity equation}

$$
\frac{\partial u}{\partial x}+\frac{\partial v}{\partial y}=0
$$

\section{Momentum equation}

$$
u \frac{\partial u}{\partial x}+v \frac{\partial u}{\partial y}=v \frac{\partial^{2} u}{\partial y^{2}}+g \beta\left(T-T_{\infty}\right)+g \beta^{*}\left(C-C_{\infty}\right)-\frac{v}{K^{1}} u
$$


Energy equation

$u \frac{\partial T}{\partial x}+v \frac{\partial T}{\partial y}=\alpha \frac{\partial^{2} T}{\partial y^{2}}+\frac{D_{m}}{c_{s}} \frac{k_{T}}{c_{p}} \frac{\partial^{2} C}{\partial y^{2}}+\frac{1}{\rho c_{p}} \frac{\partial q_{r}}{\partial y}$

\section{Concentration equation}

$u \frac{\partial C}{\partial x}+v \frac{\partial C}{\partial y}=D_{m} \frac{\partial^{2} C}{\partial y^{2}}+\frac{D_{m} k_{T}}{T_{m}} \frac{\partial^{2} T}{\partial y^{2}}-k_{1}\left(C-C_{\infty}\right)$

where $u, v$ are the velocity components in the $x$ and $y$ directions respectively, $v$ is the kinematic viscosity, $g$ is the acceleration due to gravity, $\rho$ is the density, $\beta$ is the coefficient of volume expansion, $\beta^{*}$ is the volumetric coefficient of expansion with concentration, $T, T_{w}$ and $T_{\infty}$ are the temperature of the fluid inside the thermal boundary layer, the plate temperature and the fluid temperature in the free stream, respectively,

While $C, C_{w}$ and $C_{\infty}$ are the corresponding concentrations. Also, $K^{1}$ is the permeability of a porous medium, $\alpha$ is the thermal diffusivity, $D_{m}$ is the coefficient of mass diffusivity, $c_{p}$ is the specific heat at constant pressure, $T_{m}$ is the mean fluid temperature, $k_{T}$ is the thermal diffusion ratio and $c_{s}$ is the concentration susceptibility.

The boundary conditions for the model are

$$
\begin{aligned}
& u=U_{0,} v=v_{0}, \quad \mathrm{~T}=\mathrm{T}_{w}, \mathrm{C}=\mathrm{C}_{w} \text { at } \mathrm{y}=0, \\
& u=0, \quad v=0, \quad \mathrm{~T}=\mathrm{T}_{\infty}, \quad \mathrm{C}=\mathrm{C}_{\infty} \quad \text { at } \mathrm{y} \rightarrow \infty
\end{aligned}
$$

Where $U_{0}$ is the uniform velocity and $v_{0}(x)$ is the velocity of suction at the plate.

Using the Rosseland approximation for radiation, radiative heat flux is given by Sparrow and Cess [21]

$$
q_{r}=-\frac{4 \sigma}{3 \alpha^{*}} \frac{\partial T^{4}}{\partial y}
$$

Where $\sigma$ and $\alpha^{*}$ are the Stefan-Boltzman constant and the mean absorption coeffient, respectively. We assume that the temperature differences within the flow are such that the term $T^{4}$ may be expressed as a linear function of temperature. Hence, expanding $T^{4}$ in a Taylor series about $T_{\infty}$ and neglecting higher order terms we get:

$$
T^{4} \cong 4 T_{\infty}^{3} T-3 T_{\infty}^{4}
$$

Using equations (6) and (7) equation (3) becomes:

$$
u \frac{\partial T}{\partial x}+v \frac{\partial T}{\partial y}=\alpha \frac{\partial^{2} T}{\partial y^{2}}+\frac{D_{m}}{c_{s}} \frac{k_{T}}{c_{p}} \frac{\partial^{2} C}{\partial y^{2}}+\frac{16 \sigma T_{\infty}^{3}}{3 \alpha^{*} \rho c_{p}} \frac{\partial^{2} T}{\partial y^{2}}
$$

The equations (2), (3) and (8) are coupled, parabolic and nonlinear partial differential equations and hence analytical solution is not possible. Therefore numerical technique is employed to obtain the required solution. Numerical computations are greatly facilitated by non-dimensionalization of the equations. Proceeding with the analysis, we introduce the following similarity transformations and dimensionless variables which will convert the partial differential equations from two independent variables $(x, y)$ to a system of coupled, nonlinear ordinary differential equations in a single variable $(\eta)$ i.e., coordinate normal to the plate.

In order to write the governing equations and the boundary conditions in dimensionless form, the following non-dimensional quantities are introduced. 


$$
\begin{aligned}
& \eta=y \sqrt{\frac{U_{0}}{2 v x}}, \psi=\sqrt{v x U_{0}} f(\eta), \\
& \theta(\eta)=\frac{T-T_{\infty}}{T_{w}-T_{\infty}}, \phi(\eta)=\frac{C-C_{\infty}}{C_{w}-C_{\infty}}
\end{aligned}
$$

Where $f(\eta)$ is the dimensionless stream function and $\psi$ is the dimensional stream function defined by $u=\frac{\partial \psi}{\partial x}$ and $v=-\frac{\partial \psi}{\partial y}$, just to satisfy the equation of continuity (1).

Then introducing the relation (9), into Eq.(1) we obtain

$$
u=U_{0} f^{1}(\eta) \quad \text { and } \quad v=\sqrt{\frac{v U_{0}}{2 x}}\left(\eta f^{1}-f\right)
$$

Further introducing Eqs. (9) and (10) into Eqs. (2)- (4) we respectively have

$$
\begin{aligned}
& f^{111}+f f^{11}+G_{r} \theta+G_{m} \phi-K f^{1}=0 \\
& \left(1+\frac{16}{3 R}\right) \theta^{11}+P_{r} f \theta^{1}+P_{r} D_{f} \phi^{11}=0 \\
& \phi^{11}+S_{c} f \phi^{1}+S_{c} S_{r} \theta^{11}-\gamma \phi=0
\end{aligned}
$$

Where

$G_{r}=\frac{g \beta\left(T_{w}-T_{\infty}\right) 2 x}{U_{0}^{2}}$ is the Grashof number,

$G_{m}=\frac{g \beta^{*}\left(C_{w}-C_{\infty}\right) 2 x^{2}}{v U_{0}}$ is the modified Grashof number,

$K=\frac{2 v x}{K^{1} U_{0}}$ is the permeability parameter,

$P_{r}=\frac{v}{\alpha}$ is the Prandtl number,

$S_{r}=\frac{D_{m} k_{T}\left(T_{w}-T_{\infty}\right)}{v T_{m}\left(C_{w}-C_{\infty}\right)}$ is the Soret number ,

$D_{f}=\frac{D_{m} k_{T}\left(C_{w}-C_{\infty}\right)}{c_{s} c_{p} v\left(T_{w}-T_{\infty}\right)}$ is the Dufour number,

$R=\frac{\alpha \alpha^{*} \rho c_{p}}{\sigma T_{\infty}^{3}}$ is the Radiation parameter,

$\gamma=\frac{2 k_{1} v x}{D_{m} U_{0}} \gamma$ is the Chemical reaction parameter,

$S_{c}=\frac{v}{D_{m}}$ is the Schmidt number. 
The corresponding boundary conditions are

$$
\begin{aligned}
& f=f_{w}, f^{1}=1, \theta=1, \phi=1 \text { at } \eta=0, \\
& f^{1}=0, \quad \theta=0, \quad \phi=0 \text { as } \eta \rightarrow \infty
\end{aligned}
$$

Where $f_{w}=-v_{0} \sqrt{\frac{2 x}{v U_{0}}}$ is the dimensionless suction velocity and primes denote partial differentiation with respect to the variable $\eta$.

The parameters of engineering interest for the present problem are the local skin-friction coefficient, the local Nusselt number and the local Sherwood number, which are given respectively by the following expressions Knowing the velocity field, the skin-friction at the plate can be obtained, which in non-dimensional form is given by

$\frac{1}{2} \operatorname{Re}^{\frac{1}{2}} C_{f}=f^{11}(0)$

Knowing the temperature field, the rate of heat transfer coefficient can be obtained, which in non-dimensional form, in terms of the Nusselt number, is given by

$N u(\operatorname{Re})^{-\frac{1}{2}}=-\theta^{1}(0)$,

Knowing the concentration field, the rate of mass transfer coefficient can be obtained, which in non-dimensional form, in terms of the Sherwood number, is given by

$$
\operatorname{Sh}(\mathrm{Re})^{-\frac{1}{2}}=-\phi^{1}(0)
$$

Where $\operatorname{Re}=\frac{U_{0} x}{v}$ is the Reynold's number.

\section{Mathematical Solution}

The set of coupled non-linear ordinary differential equations (11) - (13) with boundary conditions (15) have been solved numerically, by using Crack Nickels implicit finite difference method. A step size of $\Delta \eta=$ 0.01 was selected to be satisfactory for a convergence criteria of $10^{-5}$ in all cases. The value of $\eta_{\infty}$ was found to each iteration loop by the statement $\eta_{\infty}=\eta_{\infty}+\Delta \eta$. In order to see the effect of step size $\Delta \eta$ we ran the code for our model with two different step sizes $\Delta \eta=0.01, \Delta \eta=0.001$ and each case we found very good agreement between them.

\section{Results And Discussion}

During the course of discussion about the effects of various parameters on the flow field the following considerations are made:

(i) The value of Prandtl number $P_{r}$ is taken equal to 0.71 , which corresponds, physically to air.

(ii) The value of Schmidt number $S_{c}$ is chosen 0.22 , which represents hydrogen at approx.

$$
T_{m}=25^{\circ} \mathrm{c} \text { and } 1 \mathrm{~atm} \text {. }
$$

(iii) The values of Dufour number $D_{f}$ and Soret number $S_{r}$ are chosen in such a way that their product is constant provided that the mean temperature $T_{m}$ is kept constant as well.

(iv) Finally, the values of Grashof number $G_{r}$, modified Grashof number $G_{m}$, suction parameter $f_{w}$, permeability parameter $K$, Radiation parameter $R$, Chemical reaction parameter $\gamma$ are chosen arbitrarily.

As a result of the numerical calculations, the dimensionless velocity, temperature and concentration distributions for the flow under consideration are obtained and their behaviour has been discussed for variations in the governing parameters viz., the Grashof number $G_{r}$, modified Grashof number $G_{m}$, Permeability 
parameter $K$, Dufour number $D_{f}$, Soret number $S_{r}$, Suction parameter $f_{w}$, Radiation parameter $R$ and the Chemical reaction parameter $\gamma$.

\subsection{Velocity Profiles}

The effect of various parameters on the fluid velocity are illustrated in Figs.2 to 6. The effect of permeability parameter $\mathrm{K}$ on the velocity field is shown in Fig.2. The parameter $K$ as defined in Eq. (14) is inversely proportional to the actual permeability $K^{1}$ of the porous medium. An increase in $K$ will therefore increase the resistance of the porous medium (as the permeability physically becomes less with increase in $K^{1}$ ) which will tend to decelerate the flow and reduce the velocity. The effect of Grashof number $G_{r}$ on the velocity filed is presented in Fig.3. The Grashof number $G_{r}$ signifies the relative effect of the thermal buoyancy force to the viscous hydrodynamic force in the boundary layer. As $G_{r}$ increases the velocity of the fluid increases. Fig.4 presents velocity profiles in the boundary layer for various values of modified Grashof number $G_{m}$. The modified Grashof number $G_{m}$ defines the ratio of the species buoyancy force to the viscous hydrodynamic force. As $G_{m}$ increases the fluid velocity increases. From Fig.5 we observe that an increase in the suction parameter $f_{w}$ decreases the fluid velocity. The effect of Radiation parameter $R$ on the velocity field is shown in Fig.6. As the radiation parameter increases the velocity of the fluid decreases.

\subsection{Temperature profiles}

The influences of various embedded parameters on the fluid temperature are illustrated in Figs.7 to 14 . For different values of Dufour number $D_{f}$, the temperature profiles are plotted in Fig.7. The Dufour number $D_{f}$ signifies the contribution of the concentration gradients to the thermal energy flux in the flow. It is found that an increase in $D_{f}$ causes a rise in the temperature throughout the boundary layer. Fig.8 depicts the temperature profiles for different values of Soret number $S_{r}$. The Soret number $S_{r}$ defines the effects of the temperature gradients inducing significant mass diffusion effects. As the Soret number $S_{r}$ increases the temperature decreases. From Fig.9, it is observed that an increase in the permeability parameter $K$ increases the temperature of the fluid. From Fig.10, it is noticed that an increase in the Radiation parameter $R$ decreases the temperature. Figs. 11 to 13 depicts the temperature profiles for different parameters viz., Suction parameter $f_{w}$, the Grashof number $G_{r}$, modified Grashof number $G_{m}$. As $f_{w}, G_{r}$ and $G_{m}$ increases the temperature of the fluid decreases. From Fig.14, we noticed that the temperature increases with increase of Chemical reaction parameter $\gamma$.

\subsection{Concentration Profiles}

The effect of various thermo physical parameters on the fluid concentration are illustrated in Figs. 15 to 19. From Fig. 15 we noticed that, as the Soret number $S_{r}$ increases the concentration of the boundary layer also increases. For different values of Dufour parameter $D_{f}$, Suction parameter $f_{w}$ and the modified Grashof number $G_{m}$ the concentration profiles are plotted in Figs. 16 to 18. It is observed that, an increase in $D_{f}, f_{w}$ and $G_{m}$ decreases the concentration. Finally we noticed from Fig.19 that the concentration of the boundary layer decreases with increase of the chemical reaction parameter $\gamma$. 


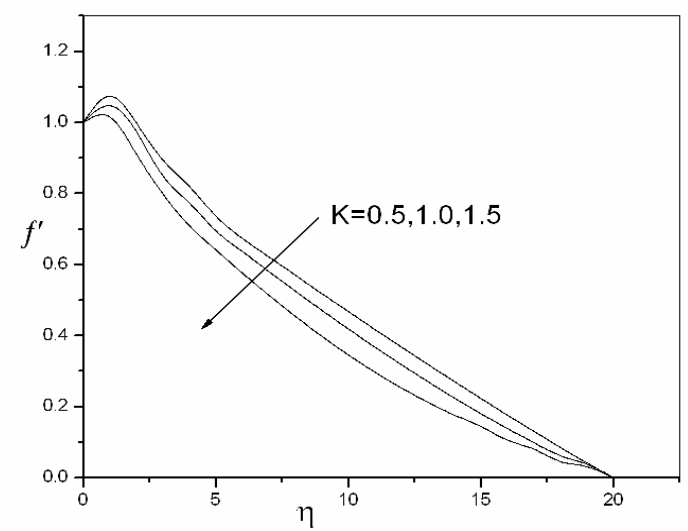

Fig. 2: Velocity profile varying with $K$ for different values of $P_{r}=0.71, S_{c}=0.22, G_{m}=4, \gamma=1$, $f_{w}=0.5, S_{r}=2.0, D_{f}=0.03, R=1, G_{r}=10$

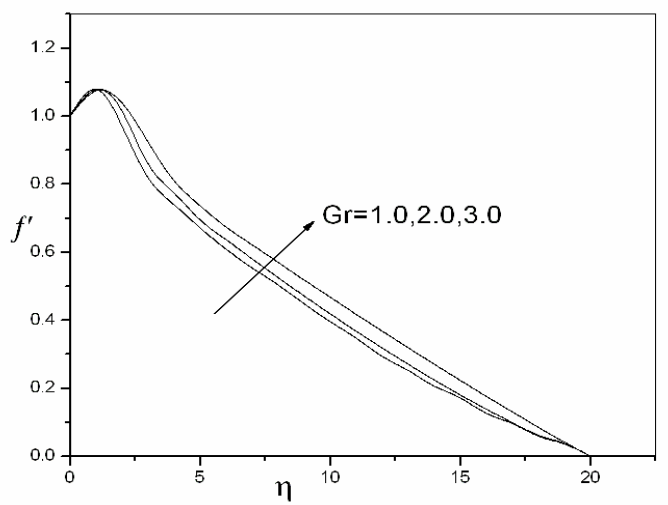

Fig 3: Velocity profile varying with $G_{r}$ for different values of $P_{r}=0.71, S_{c}=0.22, G_{m}=4, \gamma=1$, $f_{w}=0.5, S_{r}=2.0, K=1, R=1, D_{f}=0.03$

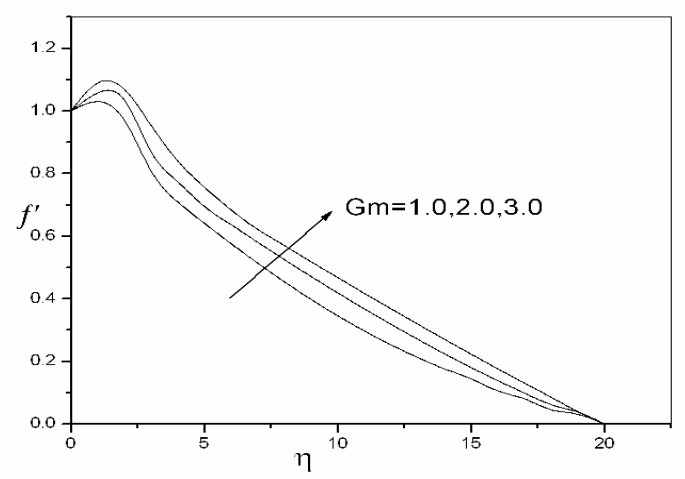

Fig 4: Velocity profile varying with $G_{m}$ for different values of $P_{r}=0.71, S_{c}=0.22, G_{r}=10, \gamma=1$, $f_{w}=0.5, S_{r}=2.0, K=1, R=1, D_{f}=0.03$ 


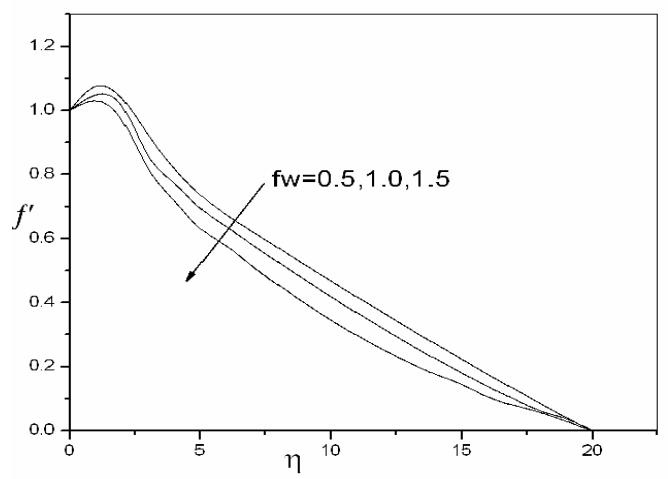

Fig 5: Velocity profile varying with $f_{w}$ for different values of $P_{r}=0.71, S_{c}=0.22, G_{r}=10, G_{m}=4$, $S_{r}=2.0, K=1, R=1, \gamma=1, D_{f}=0.03$

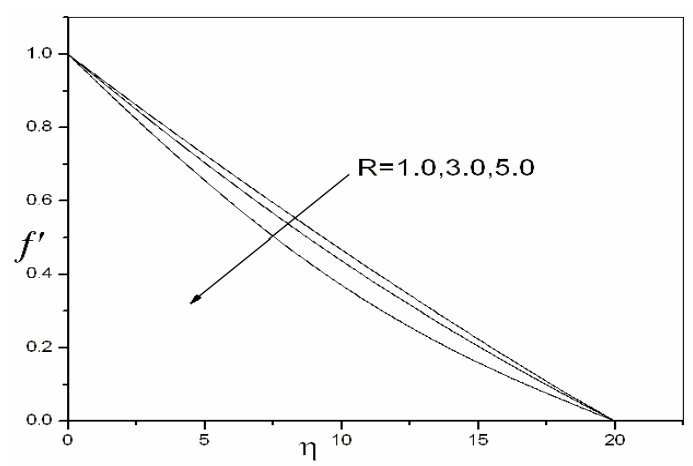

Fig 6: velocity profile varying with $R$ for different values of $P_{r}=0.71, S_{c}=0.22, G_{m}=4, \gamma=1$, $f_{w}=0.5, S_{r}=2.0, D_{f}=0.03, K=1, G_{r}=10$

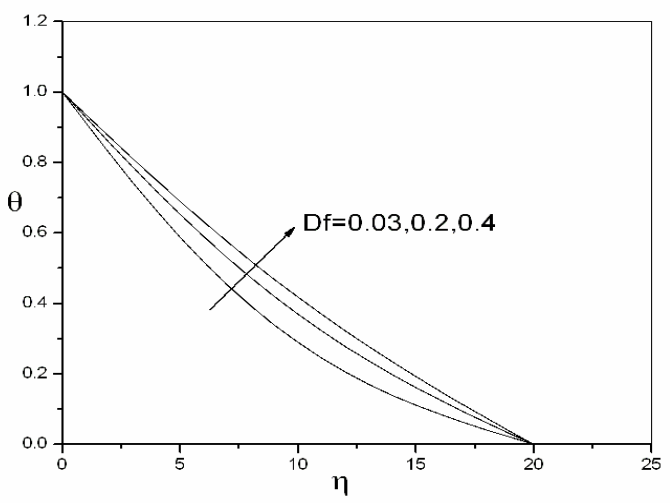

Fig 7: Temperature profile varying with $D_{f}$ for different values of $P_{r}=0.71, S_{c}=0.22, G_{m}=4$, $f_{w}=0.5, S_{r}=2.0, K=1, R=1, \gamma=1, G_{r}=10$ 


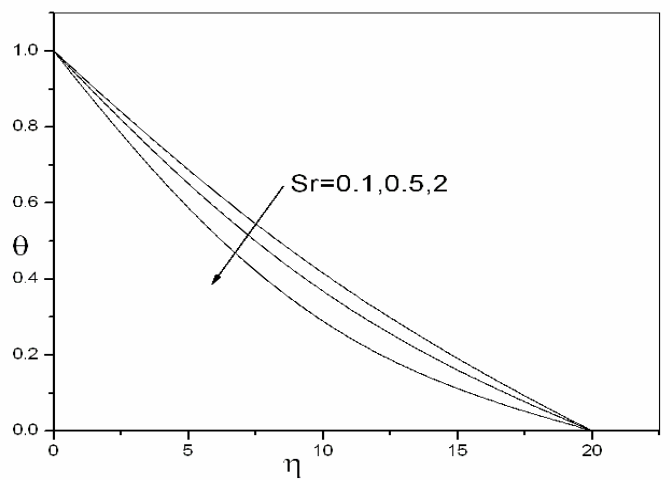

Fig 8: Temperature profile varying with $S_{r}$ for

different values of $P_{r}=0.71, S_{c}=0.22, G_{m}=4$,

$f_{w}=0.5, K=1, D_{f}=0.03, R=1, \gamma=1, G_{r}=10$

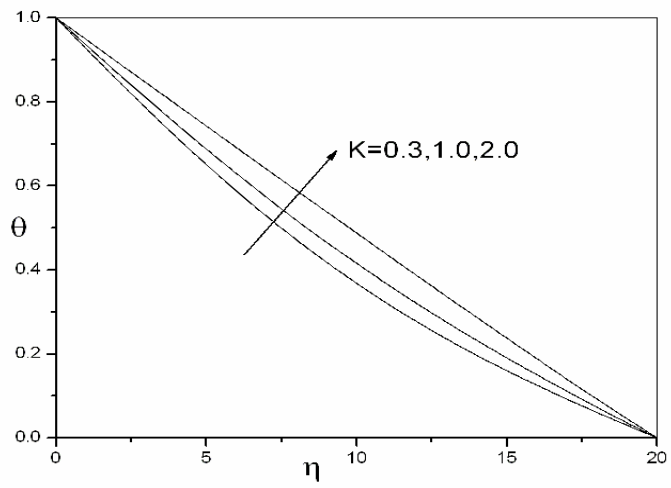

Fig 9: Temperature profile varying with $K$ for

different values of $P_{r}=0.71, S_{c}=0.22, G_{m}=4$,

$f_{w}=0.5, S_{r}=2.0, D_{f}=0.03, R=1, \gamma=1, G_{r}=10$

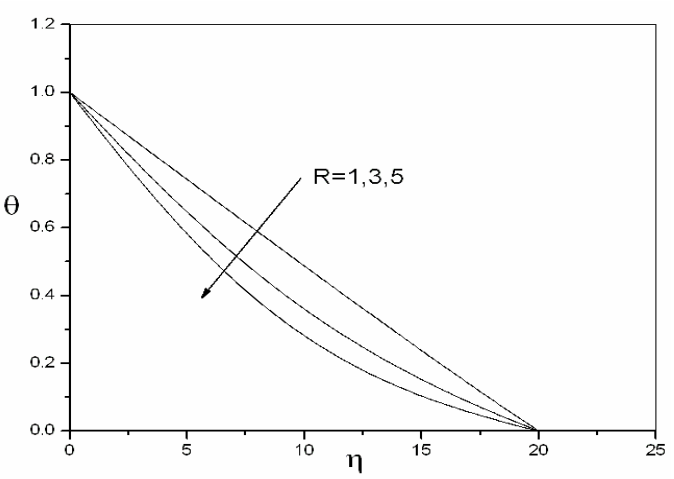

Fig 10: Temperature profile varying with $R$ for different values of $P_{r}=0.71, S_{c}=0.22, G_{m}=4$, $f_{w}=0.5, S_{r}=2.0, D_{f}=0.03, K=1, \gamma=1, G_{r}=10$ 


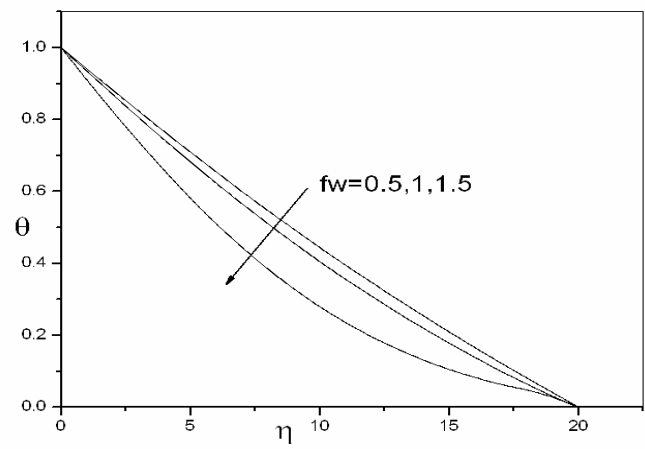

Fig 11: Temperature profile varying with $f_{w}$ for different values of $P_{r}=0.71, S_{c}=0.22, G_{r}=10$, $G_{m}=4, S_{r}=2.0, K=1, R=1, \gamma=1, D_{f}=0.03$

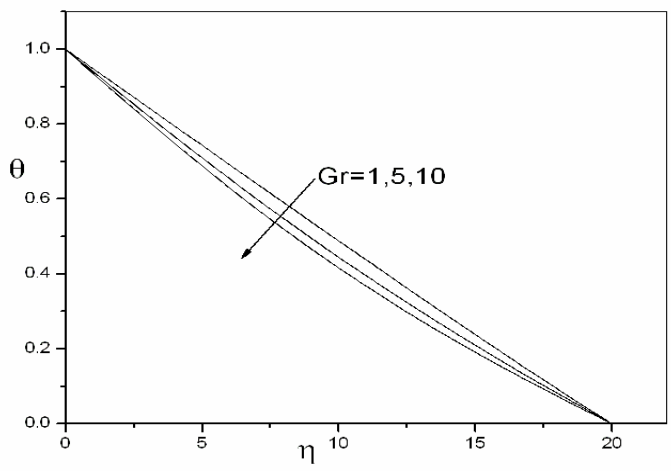

Fig 12: Temperature profile varying with $G_{r}$ for different values of $P_{r}=0.71, S_{c}=0.22, G_{m}=4$, $f_{w}=0.5, S_{r}=2.0, K=1, R=1, \gamma=1, D_{f}=0.03$

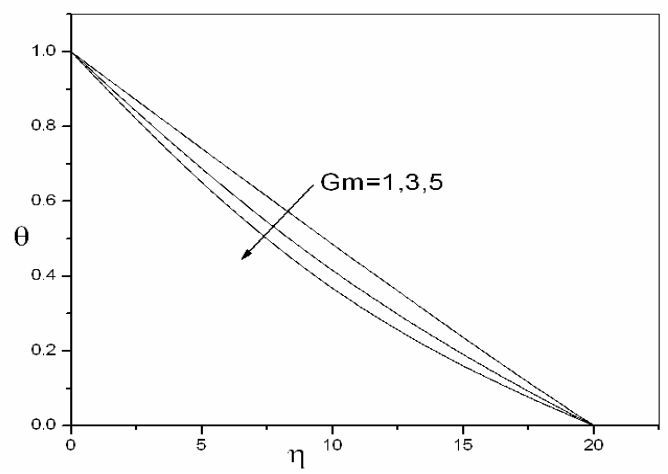

Fig 13: Temperature profile varying with $G_{m}$ for different values of $P_{r}=0.71, S_{c}=0.22, G_{r}=10$, $f_{w}=0.5, S_{r}=2.0, K=1, R=1, \gamma=1, D_{f}=0.03$ 


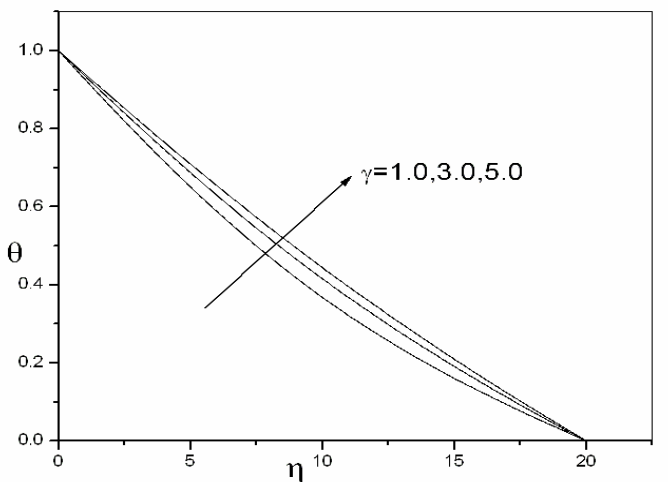

Fig 14: Temperature profile varying with $\gamma$ for different values of $P_{r}=0.71, S_{c}=0.22, K=1$ $G_{m}=4, f_{w}=0.5, S_{r}=2.0, R=1, G_{r}=10, D_{f}=0.03$

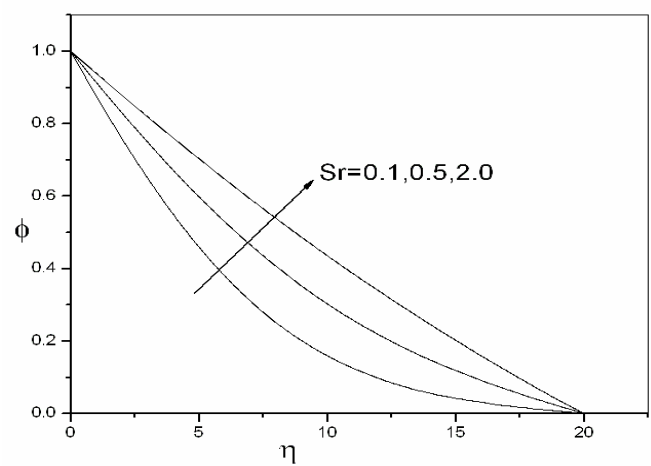

Fig 15: Concentration profile varying with $S_{r}$ for different values of $P_{r}=0.71, S_{c}=0.22, K=1$,

$G_{r}=10, G_{m}=4, f_{w}=0.5, R=1, \gamma=1, D_{f}=0.03$

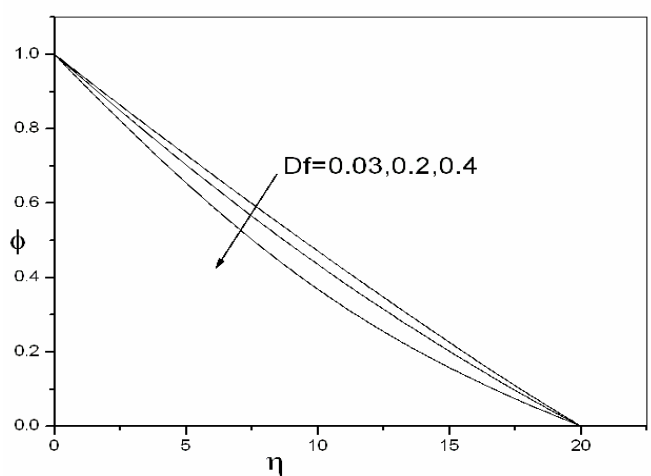

Fig 16: Concentration profile varying with $D_{f}$ for different values of $P_{r}=0.71, S_{c}=0.22, K=1$ $G_{m}=4, f_{w}=0.5, S_{r}=2.0, R=1, \gamma=1, G_{r}=10$ 


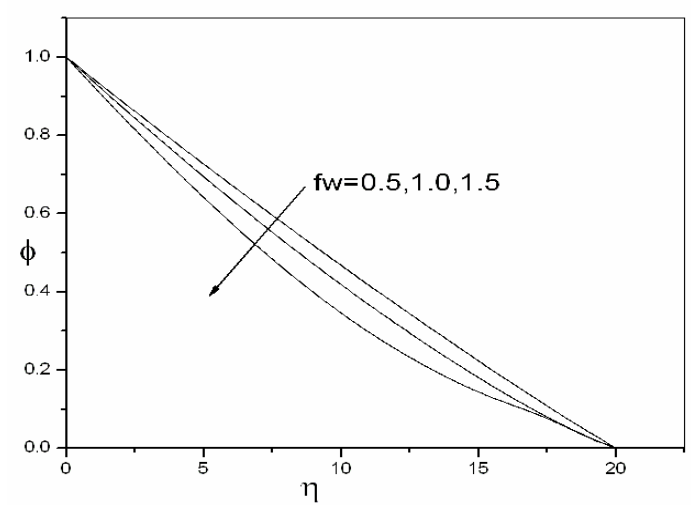

Fig 17: Concentration profile varying with $f_{w}$ for different values of $P_{r}=0.71, S_{c}=0.22, G_{r}=10$, $G_{m}=4, S_{r}=2.0, K=1, R=1, \gamma=1, D_{f}=0.03$

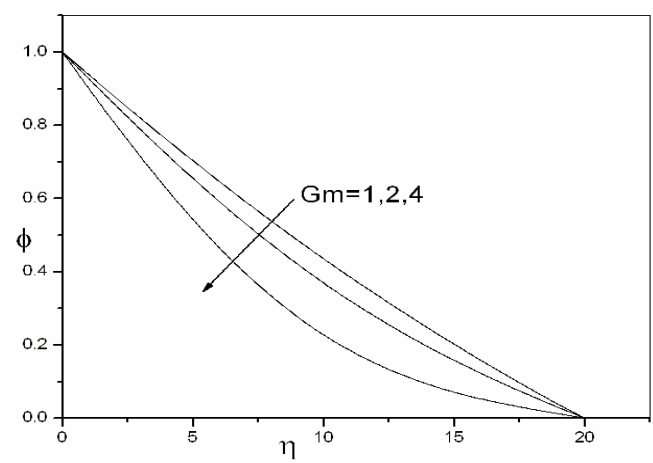

Fig 18: Concentration varying with $G_{m}$ for different of $P_{r}=0.71, S_{c}=0.22, G_{r}=10, \gamma=1$, $S_{r}=2.0, f_{w}=0.5, K=1, R=1, D_{f}=0.03$

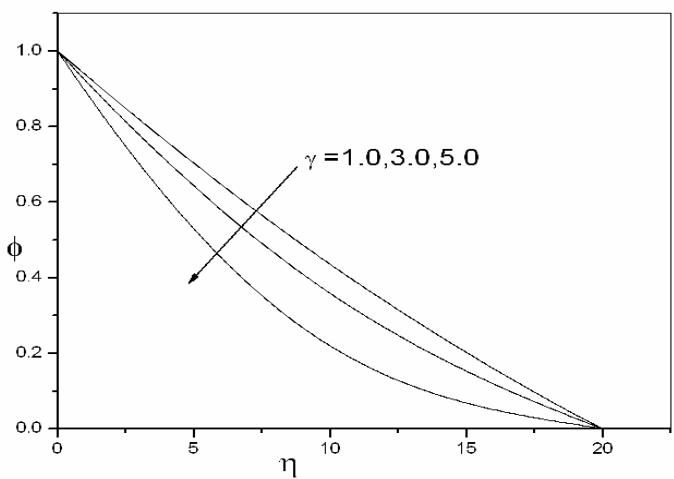

Fig 19: Concentration profile varying with $\gamma$ for different values of $P_{r}=0.71, S_{c}=0.22, K=1, R=1$, $G_{m}=4, f_{w}=0.5, S_{r}=2.0, G_{r}=10, D_{f}=0.03$. 


\section{Conclusions}

In this paper a mathematical model has been presented for the Influence of Chemical reaction on steady free convection flow past a semi-infinite moving vertical plate in a porous medium with thermal radiation. Using the similarity transformations a set of ordinary differential equations has been derived for the conservation of mass, momentum and species diffusion in the boundary layer. These nonlinear, coupled differential equations have been solved under valid boundary conditions using Implicit finite-difference method. The conclusions of the study are as follows:

1. The velocity increases with increase of Grashof number and modified Grashof number.

2. The velocity decreases with an increase in the permeability parameter.

3. The temperature and velocity of the fluid decreases with increase of Radiation parameter

4. The concentration decreases and temperature increases with increase of Chemical reaction parameter.

5. The velocity, temperature and concentration decreases with increase of Suction parameter.

6. The temperature increases with increase of Dufour number and decreases with increase of Soret number.

\section{Nomenclature}

$C$ - concentration

$c_{p}$ - specific heat at constant pressure

$c_{s}$ - concentration susceptibility

$D_{m}-$ mass diffusivity

$D_{f}$ - Dufour number

$f$ - dimensionless stream function

$f_{w}$ - dimensionless suction velocity

$g$ - acceleration due to gravity

$G_{r}$ - Grashof number

$G_{m}$ - modified Grashof number

$K$ - Permeability parameter

$k_{T}$ - Thermal diffusion ratio

$\mathrm{Nu}-$ Nusselt number

$P_{r}-$ Prandtl number

Re - Reynold's number

$S_{c}$ - Schmidt number

$\mathrm{Sh}$ - Sherwood number

$S_{r}$ - Soret number

$T$ - Temperature

$T_{m}$ - mean fluid temperature

$U_{0}$ - Uniform velocity

$u, v$-velocities in the $x$ and $y$-direction respectively

$x, y$-Cartesian coordinates along the plate and normal to it, respectively

$\alpha-$ Thermal diffusivity

$\beta$ - coefficient of thermal expansion

$\beta^{*}$ - coefficient of concentration expansion

$\rho$ - density of the fluid

$v$ - Kinematic viscosity

$\theta$-dimensionless temperature

$\phi$-dimensionless concentration

$\alpha^{*}$-mean absorption coefficient

$\sigma$-Stefen-Boltzman constant 
$R$ - Radiation parameter

$\gamma$-Chemical reaction parameter

Subscripts

$w$ - condition at wall

$\infty-$ condition at infinity

\section{REFERENCES}

[1]. D.A. Nield, A. Bejan, Convection in Porous Media (third ed., Springer, New York, 2006).

[2]. D.B. Ingham, I. Pop (Eds.), Transport Phenomena in Porous Media (Vol. III, Elsevier, Oxford, 2005).

[3]. K. Vafai (Ed.), Handbook of Porous Media (second ed., Taylor \& Francis, New York, 2005).

[4]. P. Vadasz, Emerging Topics in Heat and Mass Transfer in Porous Media (Springer, NewYork, 2008).

[5]. Anghel M., Takhar H.S. and Pop I., Dufour and Soret effects on free convection boundary- layer over a vertical surface embedded in a porous medium, Studia Universitatis Babes-Bolyai Mathematica, XLV, 2000, 11-21.

[6]. Postelnicu A., Influence of a magnetic field on heat and mass transfer by natural convection from vertical surfaces in porous media considering Soret and Dufour effects, Int. J. Heat Mass Transfer, 47, 2004, 1467-1472.

[7]. nuar Ishak, MHD Boundary layer flow due to an exponentially stretching sheet with radiation effect, Sains Malaysiana, 40(4), 2011, 391-395.

[8]. P. Bala Anki Reddy and N. Bhaskar Reddy, Thermal radiation effects on hydro-magnetic flow due to an exponentially stretching sheet, Int. J. Appl. Math. Comput., 3(4), 2011, 300-306.

[9]. V. Singh and Shweta Agarwal, Heat transfer in a second grade fluid over an exponentially stretching sheet through porous medium with thermal radiation and elastic deformation under the effect of magnetic field, Int. J. of Appl. Math and Mech., 8(4), 2012, 41-63.

[10]. Makinde, O.D., A. Ogulu, The effect of thermal radiation on the heat and mass transfer flow of a variable viscosity fluid past a vertical porous plate permeated by a transverse magnetic field, Chemical Engineering Communications, 195(12), 2008, 1575-1584.

[11]. S. Shivaiah and J. Anand Rao, Effects of Soret Dufour and thermal radiation on unsteady MHD free convection flow past an infinite vertical porous plate in the presence of chemicalReaction, Int. J. of Appl. Math and Mech, 7 (13), $2011,58-76$.

[12]. S. R. VEMPATI, A. B. LAXMI-NARAYANA-GARI, Soret and Dufour effects on unsteady MHD flow past an infinite vertical porous plate with thermal radiation, Appl. Math. Mech. -Engl. Ed, 31(12), 2010, 1481- 1496.

[13]. Shateyi, S. and Petersen, M., Thermal radiation and buoyancy effects on heat and mass tra over a semi-infinite stretching surface with suction and blowing, Journal of Applied Mathematics, 2008, 1-12 .

[14]. Chamkha, A.J., Thkhar, H.S. and Soundalgekar, V.M, Radiation effects on free convection flow past a semi- infinite vertical plate with mass transfer, Chemical Engineering Journal, 84, 2001, 335-342.

[15]. Mansour, M. A., El-Anssary, N. F., and Aly, A. M, Effects of chemical reaction and thermal stratification on MHD free convective heat and mass transfer over a vertical stretching surface embedded in a porous media considering Soret and Dufour numbers, Journal of Chemical Engineering, 145(2), 2008, 340-345.

[16]. B. Chandra and M. Kumar, The Combined Effect of Chemical Reaction, Radiation on Heat and Mass Transfer along a Continuously Moving Surface in Presence of Thermophoresis, Journal of Applied Fluid Mechanics, 6(3), 2013, $351-356$.

[17]. M.Q. Al-Odat and T.A. Al-Azab, Influence of chemical reaction on transient MHD free convection over a moving vertical plate, Emirates Journal for Engineering Research, 12 (3), 2007, 15- 21.

[18]. Ibrahim FS, Elaiw AM and Bakr AA, Effect of the chemical reaction and radiation absorption on the unsteady MHD free convection flow past a semi infinite vertical permeable moving plate with heat source and suction, Communications Nonlinear Science Numerical Simulation, 13, 2008, 1056-1066.

[19]. Kesavaiah DCh, Satyanarayana PV and Venkataramana S, Effects of the chemical reaction and radiation absorption on an unsteady MHD convective heat and mass transfer flow ast a semi-infinite vertical permeable moving plate embedded in a porous medium with heat source and suction, Int. J. of Appl. Math and Mech, 7 (1), 2011, 52-69.

[20]. M.S. Alam, M. Ferdows, M. Ota and M.A. Maleque, Dufour and Soret effects on steady free convection and mass transfer flow past a semi-infinite vertical porous plate in a porous medium, Int. J. of Applied Mechanics and Engineering,11(3), 2006, 535-545.

[21]. E.M. Sparrow, R.D. Cess, Radiation Heat Transfer (Augmented edition, Hemisphere Publishing Corp, Washington, DC, 1978). 